\title{
Experimental investigation of critical Casimir forces in binary liquid mixtures by blinking optical tweezers
} \author{
Giovanni Volpe a,e $^{\text {, }}$

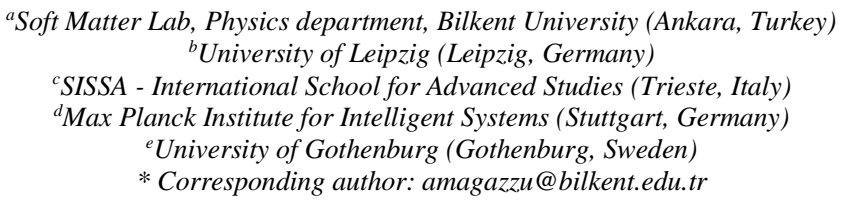

Alessandro Magazzù ${ }^{a}$, Falko Schmidt ${ }^{\mathrm{b}}$, Agnese Callegaria ${ }^{\mathrm{a}}$ Andrea Gambassi ${ }^{\mathrm{c}}$, Siegfried Dietrich ${ }^{\mathrm{d}}$,

\begin{abstract}
We investigate, for the first time and by blinking optical tweezers, the effects of critical Casimir forces (CCFs) on the free dynamics of a pair of spherical colloidal particles, immersed in binary liquid mixtures upon approaching their critical points.

OCIS codes: (350.4855) Optical tweezers or optical manipulation; (020.7010) Laser trapping; (350.4990) Particles; (020.5580) Quantum electrodynamics.
\end{abstract}

Casimir forces arise because of the quantum vacuum fluctuations of the electromagnetic field when confined between two conducting surfaces. Analogously, critical Casimir forces are due to the thermal concentration's fluctuations of a binary liquid mixture near its critical point when spatially confined between two surfaces [1-3]. The range of critical Casimir forces is strongly temperature-dependent because it is set by bulk correlation length of the binary mixture, which diverges upon approaching the critical temperature Tc [4]. Critical Casimir forces feature a universal scaling behavior, entirely determined by the internal symmetries of the system and the gross features of its interaction with the walls but independent of the properties of both.

We consider two polystyrene beads immersed in two different liquid mixtures: a water-lutidine and a waterTriton-X-100 solution near their critical points. These particles are subject to an optical and electrostatic potential as well as to critical Casimir forces when the temperature of the mixture approaches Tc. We could observe that at a sufficiently small distance, the concentration's fluctuations of the solvent are confined between the particles surfaces, modifying their interaction (Fig.1). It is noteworthy that the two solutions present different correlation lengths when approaching the critical point. Because the optical potential can screen or otherwise affect the critical Casimir forces on the particles, we used blinking optical tweezers to investigate the particles' interaction when they are not subject to the confining optical potential [5].

The effects of the CCF on the free dynamics of the two particle are showed in figure 1, where approaching the critical point of the water-lutidine mixture, attractive CCFs arise between the particle, decreasing their inter distance (Fig. 1b).
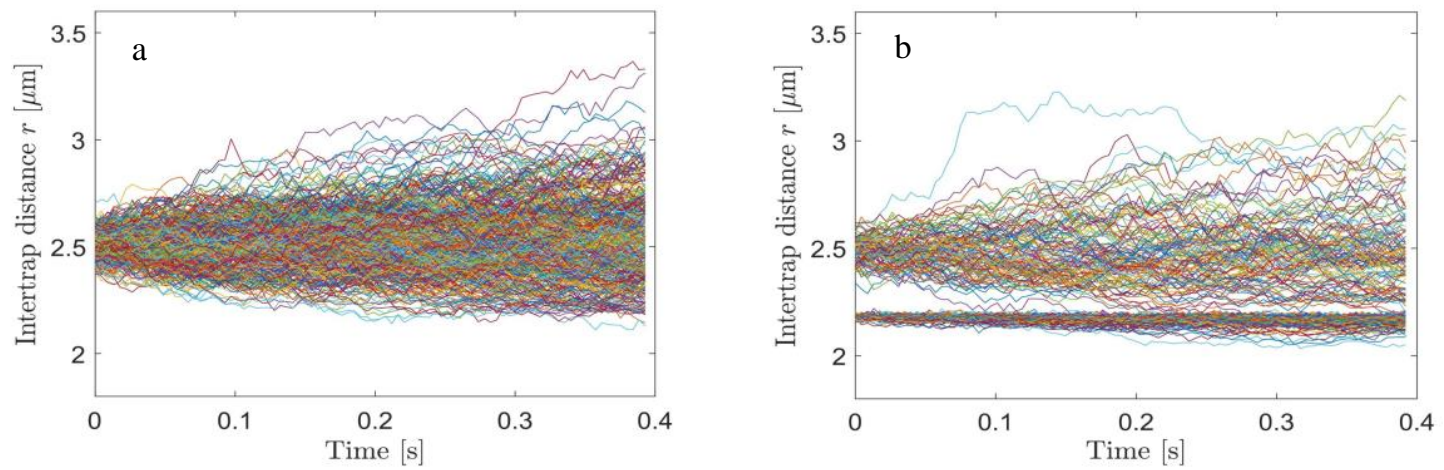

Fig.1: Time evolution of each inter-particles distance $r$ for a couple of beads immersed in a water-lutidine critical mixture; (a) time evolution of $r$ at low temperature $\left(30^{\circ} \mathrm{C}\right)$ and (b) approaching Tc, where a decreasing of $r$ is due to the occurrence of CCFs. All the inter-particle distances belonging to each temperature were obtained by video microscopy, tracking the position of the particles during all the time windows with no optical potential. 


\section{References}

[1] M. E. Fisher and P. G. D. Gennes. "Wall phenomena in a critical binary mixture." Comptes Rendus Hebdomadaires Des Seances De L Academie Des Sciences Serie B 287.8: 207-209, (1978).

[2] S. Paladugu, A. Callegari, S. Dietrich, A. Gambassi, \& Volpe, G. (2016). Nonadditivity of critical Casimir forces. Nature communications, 7 (2016).

[3] C. Hertlein, L. Helden, A. Gambassi, S. Dietrich, C. Bechinger, "Direct measurement of critical Casimir forces", Nature 451.7175: 172-175, (2008).

[4] U. Nellen, J. Dietrich, et al. "Salt-induced changes of colloidal interactions in critical mixtures", Soft Matter 7.11: 5360-5364, (2011).

[5] P. Jones, O. Maragó, and G. Volpe. Optical tweezers: Principles and applications. Cambridge University Press, (2015). 